ORIGINAL ARTICLE

\title{
Long line positioning in neonates: does computed radiography improve visibility?
}

\author{
A Evans, J Natarajan, C J Davies
}

See end of article for authors' affiliations

Correspondence to: Dr Evans, University Hospital of Wales, Heath Park, Cardiff, Wales, UK alison.evans@cardiffvale. wales.nhs.uk

Accepted 19 September 2003
Objectives: To assess the use of soft copy reporting of computed radiography (CR) images in determining intravenous long line tip position in neonates and compare visibility rates with hard copy printed images. Method: A retrospective study of all long lines inserted on the neonatal unit over a period of one year was performed. Forty five lines were inserted in 30 neonates over this time. Assessment of the CR images was made by three independent observers by reviewing the films on the viewing console and as hard copy printed films.

Results: Accurate identification of the line tip could be made in $66.7 \%$ of cases $(\kappa=0.9)$ using hard copy images and $95.6 \%$ cases $(\kappa=1.0)$ using soft copy reporting (significant difference: $p=0.002)$. The difference in percentage visibility using the two techniques was $28.9 \%$ (95\% confidence interval $10.2 \%$ to $36.7 \%)$.

Conclusion: The use of soft copy review of CR image improves the visibility of the line tip position compared with hard copy films and reduces the need for repeat radiographs with/without intravenous contrast.
$\mathrm{P}$ ercutaneously inserted intravenous long lines are often used in the routine care of patients on the neonatal unit. Importance must be paid to the correct positioning of the line, preferably with the tip lying within the superior vena cava or inferior vena cava, outside the cardiac chambers. ${ }^{1-3}$ Suboptimal or incorrect positioning can result in a variety of complications including perforation, which may lead to extravasation of intravenous fluids, pleural and peritoneal effusions, and, more seriously, pericardial effusions and cardiac tamponade. ${ }^{14-8}$ The course of the line and positioning of the tip is usually assessed using plain film radiography, traditionally using film screen combinations. The accuracy of line detection depends on a variety of factors including radiographic quality, line radio-opacity, the experience of the viewing radiologist or clinician. The introduction of computed radiography (CR) and picture archiving and storage systems (PACS) has allowed post-processing manipulation of image contrast and brightness, as well as image inversion techniques, which may optimise line visibility particularly in the case of narrow calibre poorly opaque lines.

\section{METHOD}

A retrospective study was performed over the year between January 2001 and January 2002. All neonates on the neonatal unit at our district general hospital who required a percutaneous long line during this period were included in the study. Long lines were inserted by paediatric clinicians using their standard procedure. Two standard types of lines were used: Premicath 27G (Vygon, Cirencester, UK) and Epicutaneo-cava Katheter 24G (Vygon). After insertion, a plain radiograph was taken, using the CR system, to ascertain the line position. The viewing system used is Kodak Direct View PACS version 4.2. Soft copy images are viewed on $2 \mathrm{~K}$ portrait monitors, and hard copy films are printed at true size.

Both hard and soft copy (console) images were assessed by three independent observers. The hard copy films were reviewed by observers 1 and 3, and soft copy images by observers 2 and 3. Assessment was made as to whether the line tip position could be confidently identified or not.

\section{RESULTS}

All lines could be visualised using both techniques. With the hard copy reporting technique, the line tip could be accurately identified in 30/45 cases by both observer 1 and 3. There was discrepancy of opinion on two films, but overall there was excellent statistical agreement $(\kappa=0.90)$ with both observers confidently identifying the tip in 30 cases. With the soft copy reporting technique, the line tip could be accurately identified in 43/45 cases by both observer 2 and 3, with perfect agreement $(\kappa=1.0)$. The significance of the difference was assessed using McNemars test. All combinations of observers and methods were compared. All combinations revealed a significant difference in the visibility of the line tip using the two different reporting techniques $(p=0.002)$. Overall $66.7 \%$ of line tips were confidently identified on hard copy images and $95.6 \%$ using the soft copy reporting technique. The difference in the percentage of visible line tips between the two techniques was $28.9 \%$ ( $95 \%$ confidence interval $10.2 \%$ to $36.7 \%$ ). Owing to the small numbers involved, no conclusion could be made in differentiating the two types of long line used.

\section{DISCUSSION}

Accurate positioning of intravenous long lines is important to avoid potential complications that may result from misplacement. Retrospective studies have suggested overall long line complication rates of $28-88 \%,{ }^{29}$ mechanical complication rates of $13-53 \%,{ }^{2}$ and perforation rates of $3-29 \% .{ }^{47}$ 9 Mortality resulting from perforation and cardiac tamponade has been estimated at $0.76-1 \%{ }^{4}{ }^{7}$ After reports of complications associated with right atrial tip position, and subsequent Department of Health recommendations, ${ }^{10}$ there has been a move towards positioning the line tip within the distal superior vena cava for upper limb placement and distal inferior vena cava for lower limb placement. ${ }^{136}$ To allow for

Abbreviations: $C R$, computed radiography; PACS, picture archiving and storage systems 
migration and patient movement, it is recommended that the line tip lie at least $0.5-1.0 \mathrm{~cm}$ outside the cardiac outline in premature or small infants and $1.0-2.0 \mathrm{~cm}$ outside in larger infants. ${ }^{1-3}$

The narrow calibre, poorly opaque lines used, however, may be difficult to visualise using conventional radiography. Reece et $a l^{11}$ prospectively assessed line visibility on their regional neonatal intensive care unit, and found that $50 \%$ (31/62) of patients required a repeat radiograph, with the use of intravenous contrast to clarify the position of the line and tip which was not visible on the original film. They concluded that "intravenous contrast should be routinely used in the assessment of long line position in the neonate". This policy is currently the standard protocol for imaging neonatal long lines in many centres nationally, and was adopted locally for a short period until the introduction of CR and PACS within the hospital.

The main advantage of CR and PACS over conventional radiography lies in its superior contrast resolution and the ability to allow alteration of image contrast and brightness after processing. This can be used to compensate for suboptimal exposure and improve overall image quality without the need for a repeat radiograph. The major limitation is a reduction in spatial resolution compared with conventional images, although this difference is negligible with the current high quality viewing hardware available.

At our centre a plain radiograph is performed after line insertion using CR. Neonatal films are printed as hard copy images and returned to the neonatal unit for review by clinical staff. On occasion there has been doubt over line tip position, and repeat films with contrast have been felt necessary. In many of these cases, however, review of the images using the viewing console, with the benefit of contrast and brightness windowing and image inversion has resulted in improved confidence in determining tip position and avoided the need for repeat films, with its associated radiation risk. Rarely, difficulties may arise out of hours or when radiological review is not possible, and on these occasions, a repeat film with intravenous contrast may be performed.

Review of our hard copy films revealed that the line tip could be accurately identified in 30/45 cases. Of the 15 lines that were poorly visualised, it was felt that exact information on line position was required in four cases, and repeat imaging with intravenous contrast was recommended. Visibility was significantly improved, however, when the same films were viewed on the PACS console at the time of reporting. The tip could be accurately identified in $43 / 45$ cases $(95.5 \%)$ and in neither of the two poorly visualised cases was it felt necessary to re-image, as exact determination of the tip position was unlikely to influence clinical management. Overall, 13 more lines (29\%) could be seen using soft copy rather than hard copy reporting, and repeat imaging could be avoided in four cases $(9 \%)$.

Although the overall numbers are small and there is need for further controlled trials, these findings lead us to conclude that the use of soft copy reporting of radiography images significantly improves the accuracy in determining neonatal long line position and tip, and in many cases may obviate the need for further imaging or repeated films with contrast.

This may have implications for the provision of CR/PACS access on the neonatal unit, for review by clinical teams and indeed has resulted in the installation of viewing consoles with windowing and image inversion facilities on our neonatal unit locally. Access is of particular importance out of normal working hours and when immediate radiological review may not be possible.

\section{Authors' affiliations}

A Evans, J Natarajan, C J Davies, University Hospital of Wales, Cardiff, Wales, UK

\section{REFERENCES}

1 Nowlen TT, Rosenthal GL, Johnson GL, et al. Pericardial effusion and tamponade in infants with central catheters. Pediatrics 2002;110:137-42.

2 Hogan MJ. Neonatal vascular catheters and their complications. Radiol Clin North Am 1999;37:1 109-25.

3 Darling JC, Newell SJ, Dear PRF. Placement of neonatal central venous catheter tips in the right atrium: a practice to be avoided? Arch Dis Child Fetal Neonatal Ed 2001;85:F146.

4 Leipala JA, Petaja J, Fellman V. Perforation complications of percutaneous central venous catheters in very low birthweight infants. J Paediatr Child Health 2001;37:168-71.

5 Mehta NM, Nicholl RM. Positioning of long lines. Arch Dis Child Fetal Neonatal Ed 2002;86:F68-70.

6 Bar-Joseph G, Galvis AG. Perforation of the heart by central venous catheters in infants: guidelines to diagnosis and management. J Pediatr Surg 1983;18:284-7.

7 Nadroo AM, Lin J, Green RS, et al. Death as a complication of peripherally inserted central catheters in neonates. J Pediatr 2001;138:599-601.

8 Chathas MK, Paton JB, Fisher DE. Percutaneous central venous catheterisation. Three years'experience in a neonatal intensive are unit. American Journal of Diseases in Children 1990;144:1246-50.

9 Goutail-Flaud MF, Sfez M, Berg A, et al. Central venous catheter-related complications in newborn infants: a 587-case survey. J Pediatr Surg 1991;26:645-50.

10 Department of Health Recommendations. Review of four neonatal deaths due to cardiac tamponade associated with the presence of a central venous catheter. London: DOH, June 2001.

11 Reece A, Ubhi T, Craig AR, et al. Positioning of long lines: contrast versus plain radiography. Arch Dis Child Fetal Neonatal Ed 2001;84:F129-30. 


\section{PostScript}

\section{BOOK REVIEWS}

\section{Newborn surgery, second edition}

Edited by P Puri. London: Arnold, 2003, £195, pp 976. ISBN 034076144X

Neonatologists are not always directly involved in the intensive care of neonates as surgical patients. In my own case this has led to a slightly blinkered approach. I am very familiar with perinatal stabilisation of problems such as gastroschisis, with the intensive care of infants with diaphragmatic hernias, and with the referral of infants with less acute problems. However, perioperative management, particularly of uncomplicated cases, and the mysteries of operative techniques have been beyond my reach. A book, with neonatologists within its scope, ideally with strong emphasis on presentation, embryology, and associations as well as describing surgical options, would plug a significant gap in my knowledge.

With 97 chapters, typically under 10 pages each, this book certainly has breadth of coverage. Chapters typically deal with a problem such as chylothorax, subglottic stenosis, or necrotising enterocolitis and describe the authors' perspective on management. There are numerous photographs, radiographs, and drawings in nice balance with the text. Fascinating drawings, intended to complement the "comprehensive description of operative techniques" left me wondering that such complicated operations could be so simply described. The authors are drawn from all over the world, but the book's style remains uniformly European.

The book begins with a series of chapters dedicated to general and theoretical aspects of the care of these high risk infants. These areas of overlap with standard neonatal texts are very variable and, from my perspective, also very interesting. Some could have been more up to date. It was also interesting for example to see a chapter on neonatal transport written by two paediatric surgeons rather than by neonatologists.

Some overlap is inevitable in a book like this. However, I would have preferred, for example, that either there was more embryology in each surgical chapter or a more comprehensive introductory chapter. A well written chapter on ethics, from a purely North American perspective, occupies eight pages, which is also the space given to parenteral nutrition. The five sides dedicated to respiratory management of the newborn emphasised to me the potential rewards to be reaped from closer integration of training and practice in neonatology and newborn surgery.

The chapters on surgical problems are the book's strongest area. We have found the book valuable in furthering our understanding of the problems we see on a day to day basis. Many of the lesions in question are relatively rare, which makes the superspecialist multiauthor approach most valuable. The inclusion of problems sometimes dealt with by neurosurgeons and plastic surgery specialists makes this an especially attractive volume. Only the occasional chapter seemed to focus too heavily on the authors' own experience without consideration for the variety of techniques in use.

I'm glad to say that this book is the one to plug the gaps in my knowledge. I would therefore recommend this book to fellow paediatricians, much as I would encourage surgeons and neonatologists to further develop collaboration in practice and in training.

S Oddie

Royal Victoria Infirmary, Newcastle upon Tyne NE2 2PU, UK; s.j.oddie@ncl.ac.uk

\section{Nestle nutrition workshop series: pediatric program, Vol 52: micronutrient deficiencies in the first months of life}

Edited by F M Delange, K P West Jr, S Karger. 2003, \$198.25 (hardcover). ISBN 3805575599

Micronutrient deficiencies in the first few months of life may not keep you awake at night if you are working in the UK, and this book may not grab your attention straight away, but you should give it some consideration whatever your branch or specialty in paediatrics. The book is a collection of 16 papers, written by an international panel of experts, which are the proceedings of a workshop held in Dubai in October 2002.

Most of us will be familiar with the problems of iron deficiency in early infancy and the debate on the role of neonatal vitamin $\mathrm{K}$ administration, and, if pushed, many of us would be able to say something about the public health implications of maternal folic acid supplementation and prevention of neural tube defects. This book presents papers that provide thorough state of the art reviews of these subjects. The practice of most UK based paediatricians won't frequently encompass micronutrient deficiencies outside of these aforementioned areas, but this book reminds us that, from a global perspective, nutritional deficiency problems are extremely prevalent. Vitamin A deficiency probably affects over $40 \%$ of the world's children, and iodine deficiency affects over $10 \%$, with salt iodination theoretically simple, but practically complicated. Iron deficiency is a truly global problem which affects at least one in three children world wide.

Many of us might be surprised to learn that over $50 \%$ of children in China and Tibet have features of rickets (which is also a growing (sic) problem among certain groups in the UK), and the latest evidence on the benefits of zinc supplementation in the prevention and treatment of diarrhoea, and in promotion of linear growth from field trials in developing countries, is truly compelling. Because the book is really a series of presented papers, it is genuinely more readable than a textbook on the subject. A paper on the relation between micronutrients in pregnancy and early infancy and mental and psychomotor development, and another on special micronutrient concerns in premature infants were of particular interest to my personal practice.
Discussions after the papers were presented have been included and often highlight areas of uncertainty or real practical importance.

Of course, in a book such as this there are going to be areas that don't get covered, and, if you were looking for a comprehensive tome on this subject, then spending your money on a textbook might be better. But many of us purchase textbooks and then allow them to sit on the shelves collecting dust while we only "dip into" them occasionally. The good thing about this type of book is that you might actually end up reading some of it!

N Embleton

Consultant in Neonatal Medicine, Royal Victoria Infirmary, Newcastle upon Tyne, UK; n.d.embleton@ncl.ac.uk

\section{LETTERS}

\section{Dehydration: the main cause of fever during the first week of life}

We read with interest the findings of Maayan-Metzger et al on fever in healthy newborns during the first days of life.

It is difficult to identify febrile neonates at low risk of serious bacterial infection. ${ }^{2}$ Although no consensus exists on the optimal approach to diagnosis and treatment, current guidelines recommend that febrile infants less than 28 days of age be admitted to hospital and given intravenous antibiotics for 48-72 hours. However, as mentioned in this report, dehydration is the primary cause of fever especially during the first days of life. We retrospectively reviewed the medical charts of patients admitted to our neonatal intensive care unit with fever between 1 May 1999 and 30 September 2003.

The inclusion criteria were gestational age $\geqslant 37$ weeks, 1-7 days of postnatal age excluding the first day of life, axillary or rectal temperature $\geqslant 37.8^{\circ} \mathrm{C}$ on admission, normal physical examination with well appearance, no signs of focal infection, and no history of illness or antibiotics.

Overall, 46 febrile neonates were included in the study. Most (90-95\%) were exclusively breast fed. Laboratory data included complete blood count, C reactive protein, serum urea and sodium concentrations, urinalysis, and blood, urine, and cerebrospinal fluid cultures. The mean (SD) age on admission was 3.4 (1.9) days. The mean (SD) duration of fever was 2.8 (2.4) hours. Twenty seven infants $(59 \%)$ had lost $8-24.3 \%$ of their birth weights. In 34 of the babies, white blood cell counts were between 5000 and $15000 / \mathrm{mm}^{3}$. Serum sodium concentrations were obtained in 35 patients: mean (SD) was 147 (6.7) $\mathrm{mmol} / \mathrm{l}$, and in $14(40 \%)$ the levels were equal to or higher than $150 \mathrm{mmol} / \mathrm{l}$. There was a positive correlation between weight loss and high serum sodium concentration $(\mathrm{p}=0.002)$. Mean $(\mathrm{SD})$ serum urea nitrogen concentration was 19.3 (11.1) mmol/l. In 22 (48\%) babies, serum bilirubin concentration was equal to or greater than $220 \mathrm{mmol} / \mathrm{l}$.

Cultures were positive in seven babies. Coagulase negative staphylococci were recovered from five blood cultures and considered 
to be contaminated both clinically and in a negative repeated culture. In one infant, blood culture was positive for Staphylococcus aureus, and Enterococcus grew from culture of the urine in the other. Most admissions (83\%) were between June and early October, which are the warmest months of the year in this area. In this low risk group of infants, only two patients had serious bacterial infection. Compatible with the findings of Maayan-Metzger et $a l^{1}$ the results of our study support dehydration as the main cause of fever during the first week of life. As most of our cases occurred during summer and early autumn, environmental temperature may have an additive effect in this population.

F Tiker, B Gurakan, H Kilicdag, A Tarcan Faculty of Medicine, Baskent University, Ankara, Turkey; filiztiker@yahoo.com

doi: 10.1136/adc.2003.047696

\section{References}

1 Maayan-Metzger A, Mazkereth R, Kuint J. Fever in healthy asymptomatic newborns during the first days of life. Arch Dis Child Fetal Neonatal Ed 2003:88:F312-14.

2 Baker MD, Bell LM. Unpredictability of serious bacterial illness in febrile infants from birth to 1 month of age. Arch Pediatr Adolesc Med 1999;153:508-11.

\section{Increasing incidence of moderate neonatal hyperbilirubinaemia in Wirral}

Severe neonatal jaundice and bilirubin encephalopathy have been reported with increasing frequency from North America and Europe. ${ }^{1-3}$ There are no published reports of similar trends in Britain. We therefore examined trends in moderate neonatal hyperbilirubinaemia in Wirral Hospital between 1991 and 2001. Neonates of $\geqslant 34$ weeks gestation with a serum bilirubin of $\geqslant 340 \mu \mathrm{mol} / \mathrm{l}$ were identified from the laboratory database. Trends in hyperbilirubinaemia were analysed using the $\chi^{2}$ test for trend.

A total of 184 infants were identified; 122 presented before initial discharge, and 62 were readmitted. Median (interquartile range) gestational age was 38 (37-39) weeks, and $69 \%$ of affected infants were breast fed. The incidence of moderate jaundice increased from 2.4/1000 live births in 1991 to $5.5 / 1000$ in 2001 $(\mathrm{p}<0.0001)$. Despite a progressive fall in annual births, readmissions for jaundice increased from seven in the first six years of study to 55 in the second five years $(\mathrm{p}<0.0001)$. Five infants needed exchange transfusion; all had haemolytic diseases. All were identified before initial discharge. No infants developed bilirubin encephalopathy, and none died.

Ours is the only report of recent trends in neonatal jaundice in Britain. Whether our experience is representative is not known, nor is the national incidence of bilirubin encephalopathy. These questions may be answered by this continuing study, supported by the British Paediatric Surveillance Unit, of severe neonatal jaundice.

F Walston, D Manning

Department of Paediatrics, Wirral Hospital, Merseyside CH49 5PE, UK; flossige@yahoo.com

W D Neithercut Department of Biochemistry, Wirral Hospital doi: 10.1136/adc.2003.037242
References

1 Seidman DS, Stevenson DK, Ergaz Z, et al. Hospital readmission due to neonatal hyperbilirubinaemia. Pediatrics 1995:96:727-9.

2 Maisels MJ, Newman TB. Kernicterus in otherwise healthy, breast-fed term newborns. Pediatrics 1995; $96: 730-3$

3 Ebbesen F. Recurrence of kernicterus in term and near-term infants in Denmark. Acta Paediatr 2000;89:1213-17.

\section{Use of abbreviations in daily progress notes}

Errors in medication and documentation are reported. $^{12}$ These errors, no matter how minor, could have grave consequences for the patient, especially in the paediatric population. One can imagine the potential threat to small neonates. Recently, Carroll et $a l^{3}$ described problems in residents' progress notes in a neonatal intensive care unit. Being the busiest centre in the country, managing the great majority of seriously sick neonates, we are at a very high risk of these errors. In view of this and as a screening audit, we looked at a few progress notes written on our inpatient neonates. One example of a progress note, written by a junior doctor, stated "Prem 32 WOG, F\&G, Problems: RDS, IVH II, S/P SVT, Stable on RA, TPR normal, PU, BO. Chest, CVS \& abdomen: NAD". This excessive and inappropriate use of abbreviations is alarming and disturbing. The abbreviations used denoted the following (in order of citation): weeks of gestation, feeder and grower, respiratory distress syndrome, intraventricular grade 2 haemorrhage, status post supraventricular tachycardia, room air, temperature pulse respiration, passed urine, bowel open, cardiovascular system, and no abnormality detected. This prompted us to look further into the use of abbreviations in the daily progress notes in our neonatal unit.

A cross section survey was carried out at the Special Care Baby Unit (SCBU), Royal Hospital, Muscat, on 7 October 2003. Thirty consecutive charts were reviewed. The progress notes written by seven different doctors (three registrars and four resident medical officers) were analysed for use of abbreviations. The commonly used ones were: CP (crystalline penicillin), RR (respiratory rate), HR (heart rate), BP (blood pressure), PA (per abdomen), O/E (on examination), NGT (nasogastric tube), UEl (urea and electrolyte 1), BGA (blood gas analysis), BBA (born before arrival), TPN (total parenteral nutrition), SLS (standard lipid solution), STS (standard TPN solution), D/W (discussed with), SBR (serum bilirubin), CTG (cardiotocograph), IUGR (intrauterine growth restriction), BT shunt (Blalock-Taussig shunt), TAT (trans-anastomotic tube), IVF (intravenous fluid or in vitro fertilisation), POD (postoperative day), ASD (atrial septum defect), VSD (ventricular septum defect), PDA (patent ductus arteriosus), TR (tricuspid regurgitation), L-R shunt (left to right shunt), TOF (tetralogy of Fallot), CRT (capillary refill time). One interesting note that needs separate mention was "Plan is to start $\mathrm{ABs}$ after $\mathrm{ABC}^{\prime \prime}(\mathrm{ABs}$, antibiotics; $\mathrm{ABC}$, aerobic blood culture).

We noted a high frequency of the use of abbreviations in our neonatal unit. This was a single day observation; we would expect much more in a longitudinal study. Fortunately, none of the abbreviations had resulted in erroneous interpretation, as most of the staff were used to them. However, this does not indicate that it is all right to use abbreviations. Standard abbreviations, such as VSD (ventricular septal defect) and PDA (patent ductus arteriosus), are acceptable, whereas others are not.

Documentation errors have been reported to be an increasing problem in day to day care of patients. ${ }^{45} \mathrm{~A}$ recent report described the same negligence in documentation by residents. Carroll et $\mathrm{al}^{3}$ found discrepancies in the daily progress notes written by a resident doctor in the neonatal intensive care unit. They also found that notes often contained inaccurate information and lacked pertinent information. We looked further into the situation and found extensive use of abbreviations in progress notes.

Our observation is not unique and requires rectification. The solution could be to standardise or eliminate the use of abbreviations in the unit. Total elimination would be difficult, as many of the abbreviations are acceptable. Thus, the use of unacceptable abbreviations should be discouraged. New medical officers should be given brief instruction on the writing of appropriate progress notes. An alternative is to use the electronic information system for all medical transcription including progress notes, as described elsewhere. $^{6}$

In conclusion, care of neonates requires good documentation of day to day progress. The use of unacceptable abbreviations should be discouraged. A follow up audit is warranted to look further into the effect and success of our recommendations.

S Manzar, A K Nair, M Govind Pai, S Al-Khusaiby

Special Care Baby Unit, Royal Hospital, PO Box 1331, Postal Code 111, Muscat, Sultanate of Oman; shabihman@hotmail.com

doi: 10.1136/adc.2003.045591

\section{References}

1 Brennan TA, Leape LL, Laird NM, et al. Incidence of adverse events and negligence in hospitalized patients. Results of the Harvard Medical Practice Study I. N Engl J Med 1991;324:370-6.

2 Cradock J, Young AS, Sullivan G. The accuracy of medical record documentation in schizophrenia. J Behav Health Serv Res 2001;28:456-65.

3 Carroll AE, Tarczy-Hornoch P, O'Reilly E, et al. Resident documentation discrepancies in a neonatal intensive care unit. Pediatrics 2003; 111:976-80

4 Bedell SE, Deitz DC, Leeman D, et al. Incidence and characteristics of preventable iatrogenic cardiac arrests. JAMA 1991;265:2815-20

5 Lester H, Tritter JQ. Medical error: a discussion of the medical construction of error and suggestions for reforms of medical education to decrease error. Med Educ 2001;35:855-61.

6 Kaushal R, Barker KN, Bates DW. How can information technology improve patients' safety and reduce medication errors in child health care. Arch Pediatr Adolesc Med 2001; 155:1002-7.

7 Menke JA, Broner CN, Campbell DY, et al. Computerized documentation system in pediatric intensive care unit. BMC Med Inform Decis Mak 2001;1:3.

\section{Use of nasal continuous positive airway pressure during neonatal transfer}

Within neonatal intensive care units, nasal continuous positive airway pressure (nCPAP) 
provides a means of respiratory support in a variety of acute and chronic clinical situations. ${ }^{12}$ We have used it as a means of respiratory support during neonatal transfer and describe our experience below.

nCPAP was provided by the Infant Flow Driver (Electro Medical Equipment Ltd, Brighton, Sussex, UK). This was clamped on to the vertical frame of the transport incubator, and a modified ventilation circuit, designed by the medical physics department of the Princess Royal Maternity, connected the Infant Flow Driver to the infant via short binasal prongs (Electro Medical Equipment Ltd). All infants were transferred by road in the West of Scotland Region dedicated neonatal ambulance. This ambulance provided an oxygen and air supply of 4000 litres each and AC power from a petrol generator.

Over a one year period from April 2002 until April 2003 there were seven nCPAP transfers involving six infants. The median gestational age at birth was 29 weeks (range 26-32) and the median age at transfer was 23 days (range 5 hours to 91 days). These included infants with complex congenital abnormalities requiring specialist treatment and those returning to their base hospital. The median transfer time was 45 minutes (range 30-60). No major problems were encountered during transfer. All transfers using nCPAP were discussed in advance with a senior neonatologist experienced in neonatal transport.

We have shown in a small and carefully selected cohort of infants that transfer with nCPAP support is feasible and safe. Our infants, with one exception, had been stable on nCPAP for some time before transfer. Further studies are required to explore whether this form of respiratory support has a role in the transfer of neonates with acute respiratory distress syndrome who are stable on nCPAP, and who would currently be intubated only because of the need for transfer.

Correct attachment of the nCPAP driver to the transport incubator system is vital. Further modifications are being engineered to our transport incubator system to comply with regulations ensuring safety in crash situations.

Even with our confidence in the use of nCPAP for selected clinical situations in transport, we would still strongly recommend that intubation remains the first choice for airway management during neonatal transfer.

J H Simpson, I Ahmed, J McLaren, C H Skeoch Princess Royal Maternity, 16 Alexandra Parade, Glasgow G32 2ER, Scotland, UK; Charles.Skeoch@northglasgow.scot.nhs.uk

doi: 10.1136/adc. 2003.033159

\section{References}

1 Jeena $\mathbf{P}$, Pillay $P$, Adhikari M. Nasal CPAP in newborns with acute respiratory failure. Ann Trop Paediatr 2002;22:201-7.

2 Davis PG, Henderson-Smart DJ. Nasal continuous positive airways pressure immediately after extubation for preventing morbidity in preterm infants. Cochrane Database Syst Rev 2000;(3):CD000143.

\section{What is the normal range of blood glucose concentration in healthy term newborns?}

The report by Dr Nicholl on "normal blood glucose concentrations in healthy term newborns" ${ }^{\prime 1}$ raises the interesting and important question of how normoglycaemia in newborns can be defined. In a comprehensive review of the literature in 1997, an expert panel of the World Health Organization concluded that there are numerous approaches to defining normoglycaemia, including the statistical approach (which was taken by Dr Nicholl), the metabolic approach (what is the concentration of blood glucose at which normal cell homoeostasis is maintained?), the neurophysiological approach (below what concentration of blood glucose does impairment of neurological functions occur?), and, perhaps most importantly, the neurodevelopmental approach (does a relation exist between neonatal blood glucose concentrations and the neurodevelopmental outcome of children years later?). ${ }^{2}$ These different approaches towards definition of normoglycaemia contribute to the controversy that surrounds this issue. ${ }^{23}$ Other factors that influence newborn blood glucose concentrations, even in healthy term newborns, are perinatal complications, ${ }^{4}$ mode of delivery, ${ }^{5}$ and feeding behaviour. ${ }^{6}$ It appears therefore that there is very little solid evidence on which judgment of neonatal blood glucose concentrations can be based. Follow up studies looking at neurodevelopmental outcome of neonatal "hypoglycaemia" (and its treatment) in healthy term infants of various delivery modes and birth weights are urgently needed.

P L P Brand

Isala klinieken, Zwolle, the Netherlands doi: $10.1136 /$ adc. 2003.035196

\section{References}

1 Nicholl OR. What is the normal range of blood glucose concentrations in healthy term newborns? Arch Dis Child 2003;88:238-9.

2 World Health Organization. Hypoglycaemia of the newborn. Review of the literature. Geneva: World Health Organization, 1997, WHO/CHD/ 97.1. www.who.int.

3 Cornblath $M$, Hawdon JM, Williams AF, et al. Controversies regarding definition of neonatal hypoglycemia: suggested operational thresholds. Pediatrics 2000;105:1141-5.

4 Schaefer-Graf UM, Rossi R, Bührer C, et al. Rate and risk factors of hypoglycemia in large-forgestational-age newborn infants of nondiabetic mothers. Am J Obstet Gynecol 2002; 187:913-17.

5 Cole MD, Peevy K. Hypoglycemia in normal neonates appropriate for gestational age. J Perinatol 1994;14:1 18-20.

6 Heck $\amalg$, Erenberg A. Serum glucose levels in term neonates during the first 48 hours of life. J Pediatr 1987;110:119-22.

\section{Gastric perforation and transillumination}

We read with interest the article of Farrugia and colleagues ${ }^{1}$ about neonatal gastrointestinal perforation. However, there was no mention of:

- Isolated gastric perforation as a cause of neonatal gut perforation, or

- Transillumination as a simple diagnostic tool of pneumoperitoneum.

We highlight these two points relating to a recent case. A 29 week gestation baby girl was born by vaginal delivery. She initially required conventional ventilation for her lung

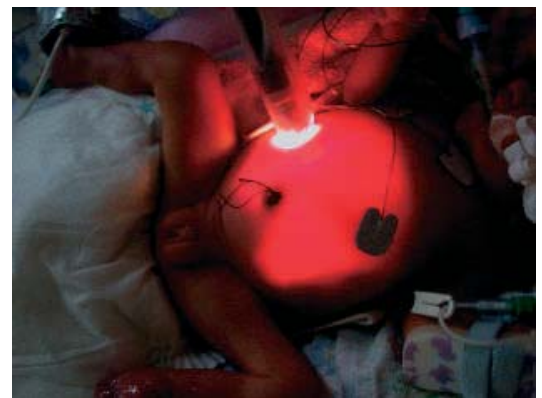

Figure 1 Transillumination of the abdomen showing pneumoperitoneum.

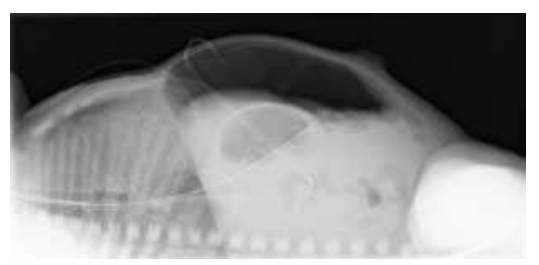

Figure 2 Abdominal radiograph confirming pneumoperitoneum.

disease. An umbilical arterial catheter was inserted but removed after a few hours due to duskiness of the toes. On day 2 she was extubated and nCPAP was tried. After a few hours, her condition deteriorated and she returned to conventional ventilation. On day 4, she was started on enteral feeding, using small volumes of breast milk, but had mild abdominal distension and some aspirates. Feeding was stopped. Her abdomen deteriorated and she had persistent metabolic acidosis. Transillumination of her abdomen was positive (fig 1) for pneumoperitoneum and was confirmed by abdominal $x$ ray examination (fig 2). At laparotomy, two small gastric perforations were identified with local areas of infarction. These were oversewn, with excellent results.

Neonatal gastric perforation is unusual but serious. Various causative factors, including prematurity and nCPAP, have been suggested. $^{2}$ Both of these were present in our case. It is also possible that emboli from the umbilical catheter led to small areas of infarction of the stomach wall.

Transillumination is a quick and easy technique for diagnosing pneumoperitoneum, ${ }^{3}$ and obviates the need for frequent radiographs.

W El-Matary, I Barnard, D Cameron Neonatal Unit, Glan Clwyd Hospital, Denbighshire LL17 ODF, UK; DrDuncan.Cameron@ cd-tr.wales.nhs.uk

doi: 10.1136/adc.2003.037507

\section{References}

1 Farrugia MK, Morgan AS, McHugh, et al. Neonatal gastrointestinal perforation. Arch Dis Child Fetal Neonatal Ed 2003;88:F75.

2 Leone RJ, Krasna IH. "Spontaneous" gastric perforation: is it really spontaneous? J Pediatr Surg 2000;35:1066-9.

3 Wyman ML, Kuhns LR. Pneumoperitoneum demonstrated by transillumination. Am J Dis Child 1976;130:1237. 


\section{CORRECTIONS}

doi: 10.1136/adc.2002.021147corr 1

Gupta A, Patel R, and Dyke M. Cost effective use of blood satellite packs in neonates: importance of birth weight. Arch Dis Child Fetal Neonatal Ed 2004;89:182-3. Dr Dyke's and Dr Gupta's affiliation was incorrectly listed as Derriford Hospital, Plymouth, Devon, UK. The authors would like to point out that their work was initially carried out at the Norfolk and Norwich (University) Hospital, Norwich, UK.

doi: 10.1136/adc.2003.030460corrl

A Evans, J Natarajan, C J Davies. Long line positioning in neonates: does computed radiography improve visibility? (Arch Dis Child Fetal Neonatal Ed 2004:89:F44-5). The authors would like to correct the author affiliations at the end of this article. Dr A Evans works at University Hospital of Wales, Cardiff, Wales, UK, and Drs J
Natarajan and C J Davies work at the Royal Glamorgan Hospital, Llantrisant, Wales, UK.

doi: 10.1136/adc.2002.020735corr 1

J Daniels, F Craig, R Wajed and M Meates. Umbilical granulomas: a randomised controlled trial (Arch Dis Child Fetal Neonatal Ed 2003;88:F257). The page number provided in the reference of this letter is incorrect and should be F431-4.

\section{IMAGES IN NEONATAL MEDICINE.}

\section{Renal fungal ball}

$\mathrm{P}$ reterm infants are prone to funga infections because of immaturity of their host defence systems (immunology and skin). Other risk factors include multiple antibiotic therapy, prolonged use of umbilical or percutaneous catheters, total parenteral nutrition, colonisation and/or past mucocutaneous candidiasis, low birth weight, endotracheal tube placement, and congenital malformation.

Common sites for invasive candidiasis are the renal system, eyes, brain, and heart Diagnostic tests should include blood and urine cultures, renal ultrasound, ophthalmological assessment, cardiac ultrasound, and examination of cerebrospinal fluid.

Candiduria may indicate colonisation, but the presence of other clinical signs increases the risk of invasive candidiasis. Fungal ball is the commonest presentation of renal fungal disease. ${ }^{1}$ Clinical presentation may vary and can be obstructive, or non-obstructive, with renal failure.

A baby born at 28 weeks gestation was known to be colonised with Candida spp in the first weeks of life. The mother had declined routine antenatal care. The baby was ventilator dependent, with umbilical lines and received multiple broad spectrum antibiotics for possible bacterial sepsis.

After one month the baby developed thrombocytopenia and renal impairment. A renal ultrasound confirmed the presence of a solitary kidney with an echogenic mass.

Limited postmortem examination revealed multiple abscesses in the renal parenchyma, which grew Candida albicans only.

Invasive fungal infections in very low birthweight babies are currently the subject of a BPSU study (http://bpsu.inopsu.com/ current.htm\#Invasive).

M Wimalendra

A Reece

R M Nicholl

Neonatal Unit, Northwick Park Hospital, North West London Hospitals NHS Trust, Harrow HAl 3UJ, UK; Richard.nicholl@nwlh.nhs.uk

\section{REFERENCE}

1 Bryant K, Maxfield C, Rabalais G. Renal candidiasis in neonates with candiduria. Pediatr Infect Dis J 1999;18:959-63.

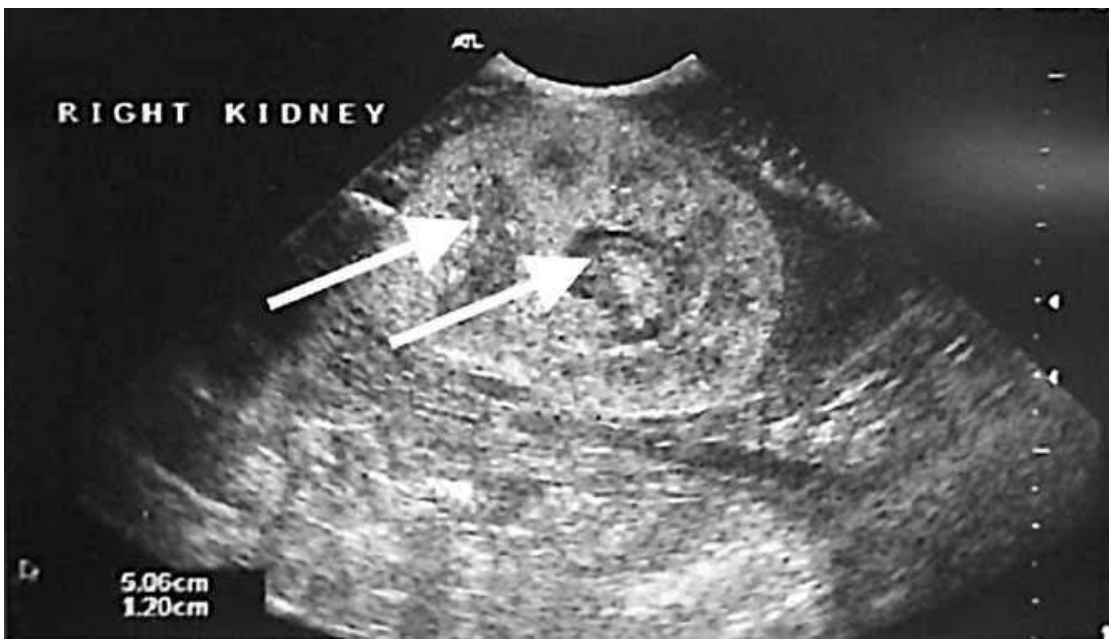

Figure 1 Solitary kidney with echodensities.

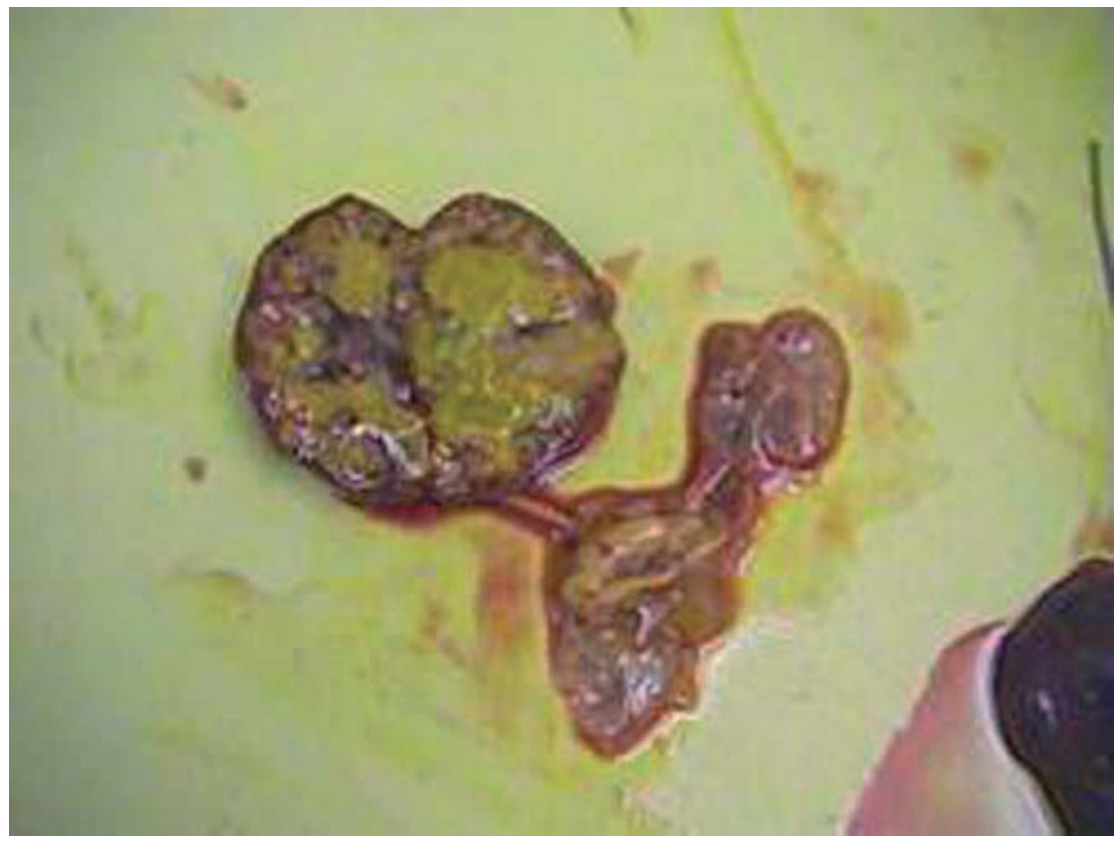

Figure 2 Gross pathology, showing multiple fungal abscesses. 